\title{
Extent-based incremental identification of reaction systems using concentration and calorimetric measurements
}

\author{
Sriniketh Srinivasan, Julien Billeter*, Dominique Bonvin \\ Laboratoire d'Automatique, Ecole Polytechnique Fédérale de Lausanne, Switzerland
}

\section{H I G H L I G H T S}

- Extent-based incremental identification models extents computed from concentrations.

- Calorimetry is related to extents of reaction and mass transfer via enthalpies.

- Extents are computed by augmenting rank-deficient concentrations with calorimetry.

- Extents computed from full-rank conc. allow estimating enthalpies from calorimetry.

- These concepts are illustrated via homogeneous and gas-liquid reaction systems.

\section{A R T I C L E I N F O}

\section{Article history:}

Available online 21 July 2012

\section{Keywords:}

Reaction kinetics

Mass-transfer rates

Extents of reaction

Extents of mass transfer

Incremental identification

Calorimetry

\begin{abstract}
A B S T R A C T
Extent-based incremental identification uses the concept of extents and the integral method of parameter estimation to identify reaction kinetics from concentration measurements. The approach is rather general and can be applied to open both homogeneous and gas-liquid reaction systems. This study proposes to incorporate calorimetric measurements into the extent-based identification approach for two main purposes: (i) to be able to compute the extents in certain cases when only a subset of the concentrations is measured and (ii) to estimate the enthalpies when all concentrations are measured. The two approaches are illustrated via the simulation of a homogeneous and a gas-liquid reaction system, respectively.
\end{abstract}

(ㄷ) 2012 Elsevier B.V. All rights reserved.

\section{Introduction}

Modeling reaction systems is essential for the monitoring, control and optimization of industrial chemical processes. The models are often first-principles models that describe the evolution of the states (concentrations, temperature and volume) by means of conservation equations of differential nature. The identification of rate laws represents the main challenge in building first-principles models for reaction systems. This is especially true in the case of heterogeneous reaction systems because of the direct coupling between the chemical reactions and the mass transfers between phases.

The identification task can be performed in one step via a simultaneous approach, or over several steps via an incremental approach, as discussed next.

In the simultaneous approach, a candidate model that includes the rate laws for all reactions and mass transfers is postulated,

\footnotetext{
* Corresponding author. Tel.: +41 2169366 99; fax: +41 216932574 .

E-mail addresses: sriniketh.srinivasan@epfl.ch (S. Srinivasan), julien.billeter@ epfl.ch (J. Billeter), dominique.bonvin@epfl.ch (D. Bonvin).
}

and the corresponding rate parameters are estimated by comparing model predictions and measured concentrations. Issues like parameter and structural identifiability [1] and experimental planning [2] are important to guarantee parameter estimates with little correlation and narrow confidence intervals. The main advantage of simultaneous identification is that it leads to optimal parameters in the maximum-likelihood sense [3]. However, simultaneous identification can be computationally costly when several candidates are available for each rate law. Furthermore, structural mismatch in one part of the model will typically result in errors in all the estimated parameters. Finally, it is often difficult to choose suitable initial guesses, which may lead to convergence problems [4].

In the incremental approach, the identification task is decomposed into a set of subproblems, for which the number of model candidates can be kept low [5]. The incremental approach usually proceeds in two steps: (1) computation of the contribution associated with each reaction and mass transfer as rates or extents and (2) individual identification of the rate laws and corresponding parameters from the computed rates or extents [6]. Due to the decoupling of the various rates or extents, each reaction and each 


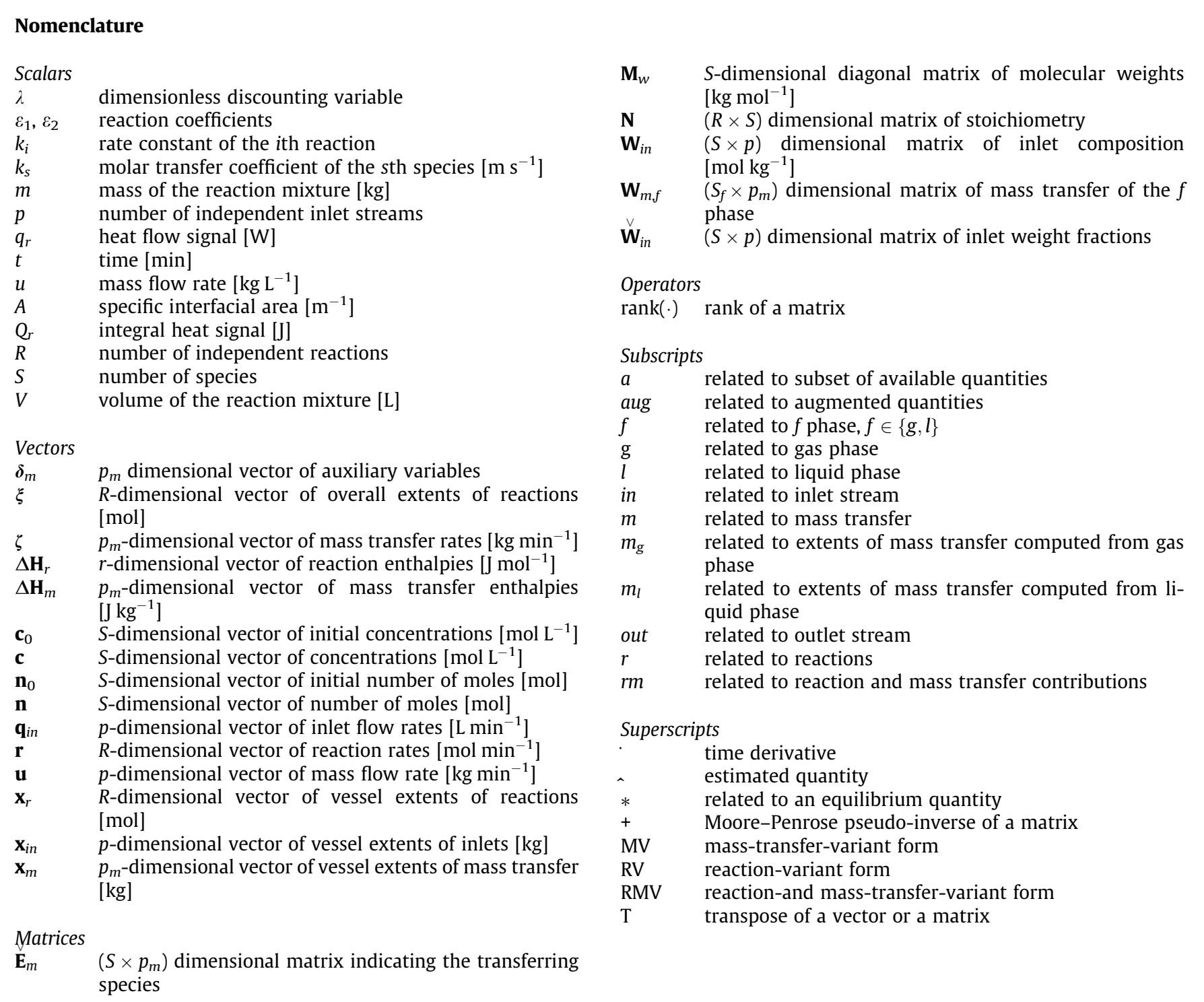

mass transfer can be dealt with individually, which avoids the combinatorial increase in the number of rate scenarios to be tested. Consequently, the computation time is also reduced significantly compared to simultaneous approach. This decoupling removes the correlation between the reaction and mass transfer parameters. Note that the computation of the rates or extents from measured concentrations is done for a given time instant without the use of a rate law to process data over time. Such an instantaneous reconstruction requires that there are at least as many measurements as there are rates or extents to be computed. This approach is also sometimes referred to as "individual identification".

Two different incremental methods have been proposed in the literature and are briefly summarized next.

In the differential method, reaction and mass transfer rates are first computed through differentiation of measured concentration profiles, without explicit knowledge of the rate laws [7]. Then, candidate rate laws are postulated for each rate profile and the corresponding parameters are estimated by comparing predicted and computed rates. The issue with the differentiation step is that it introduces a bias in the rate estimation, with the result that the rate parameters will not be statistically optimal [8]. Hence, in order to obtain statistically valid parameters, simultaneous identification is often applied as a final step using the model structure identified via the incremental approach.

In the integral method, which is considered in this work, the contribution of the reactions and mass transfers are extracted from the measured concentration profiles in the form of extents, and this without differentiation nor knowledge of the rate laws $[9,10]$. In a second step, candidate rate laws are integrated to predict the computed extents, which allows estimating the rate parameters via a least-squares problem. Because this method uses extents rather than rates, it has been termed "extent-based incremental identification". Although computationally more intensive than the differential method, the extent-based method has the advantage of computing rate parameters that are optimal in the maximum-likelihood sense.

Chemical reactions are accompanied by heat generation (consumption) in the case of exothermic (endothermic) reactions. Calorimetry is a very useful measurement technique to evaluate the power produced (consumed) by reactions. At the laboratory stage, calorimetric data can be measured at the micro scale (micrograms up to milligrams) or small scale (grams up to kilograms). At the micro scale, calorimetry is performed in thermal analytical devices, such as high-pressure micro-calorimeters, isothermal titration 
calorimeters or differential scanning calorimeters [11]. These calorimetric devices operate at conditions that differ significantly from those encountered at the production stage. Because of the small size of the measurement cell, these calorimeters do not usually allow simultaneous measurement of concentrations. At the small scale, calorimetry is performed in reaction calorimeters that are designed to mimic the operational conditions in large industrial reactors [12]. Reaction calorimeters can be combined with online or offline analytical techniques that measure the concentration of reactive species over time [13]. At the industrial scale, calorimetry can be measured in large-scale reactors by means of heat balances, dimensionless correlations and calibrations [14].

In this study, calorimetric measurements are added to concentration measurements in the framework of extent-based incremental identification. In this context, calorimetric measurements can serve two purposes. With fewer measurements than the number of extents to be computed, calorimetric measurements can be used to augment by one the rank of the measurement equations. This rank augmentation is only possible if all enthalpies are known. Alternatively, if the number of measurements is sufficient to estimate all extents, calorimetric measurements can be used to estimate the enthalpies of reaction and mass transfer, which represent valuable quantities in the context of thermal process safety and process development $[15,16]$.

The paper is organized as follows. In the Section 2, the extentbased incremental approach is briefly reviewed. Then, the use of calorimetric measurements for the purposes of rank augmentation and enthalpy estimation is discussed. In the Section 3, the use of calorimetric data is illustrated via the simulation of two reaction systems, namely, the acetoacetylation of pyrrole in a homogeneous reactor and the chlorination of butanoic acid in a gas-liquid reactor. In the Section 4, conclusions are drawn regarding the usefulness of calorimetric data.

\section{Extent-based Incremental Identification}

The extent-based incremental identification proceeds in two steps. The vector of numbers of moles is first transformed to extents and invariants and then these extents are used to identify individually the rate laws and the corresponding parameters. In this section, the differential mole balance equations are introduced and the method of extent-based incremental identification is briefly reviewed for the cases of homogeneous and gas-liquid reaction systems. Then, the inclusion of calorimetric data into this method is presented.

\subsection{Mole balance equations}

\subsubsection{Homogeneous reaction systems}

The differential mole balance equations for homogeneous reaction systems consisting of $S$ species, $R$ reactions, $p$ inlet streams and one outlet stream can be written as follows:

$\dot{\mathbf{n}}=\mathbf{N}^{\mathrm{T}} V(t) \mathbf{r}(t)+\mathbf{W}_{\text {in }} \mathbf{u}_{\text {in }}(t)-\frac{u_{\text {out }}(t)}{m(t)} \mathbf{n}(t), \quad \mathbf{n}(0)=\mathbf{n}_{0}$

where $\mathbf{n}$ is the $S$-dimensional vector of numbers of moles, $\mathbf{r}$ the $R$-dimensional vector of reaction rates, $\mathbf{u}_{i n}$ the $p$-dimensional inlet mass flow rate vector, $u_{\text {out }}$ the outlet mass flow rate, $V$ and $m$ the volume and the mass of the reaction mixture, $\mathbf{N}$ the $R \times S$ stoichi-

ometric matrix, $\mathbf{W}_{\text {in }}=\mathbf{M}_{w}^{-1} \mathbf{W}_{\text {in }}$ the $S \times p$ inlet-composition matrix with $\mathbf{M}_{w}$ the $S$-dimensional diagonal matrix of molecular weights and $\mathbf{W}_{\text {in }}=\left[\breve{\mathbf{w}}_{i n}^{1}, \ldots, \mathbf{w}_{i n}^{p}\right]$ with $\breve{\mathbf{w}}_{\text {in }}^{k}$ being the $S$-dimensional vector of weight fractions of the $k$ th inlet stream, and $\mathbf{n}_{\mathbf{0}}$ the $S$ dimensional vector of initial numbers of moles.

\subsubsection{Gas-liquid reaction systems}

For gas-liquid reaction systems, let the reactor contain the gas phase $G$ comprised of $S_{g}$ species and the liquid phase $L$ composed of $S_{l}$ species. The two phases are connected by $p_{m}$ mass transfers for the $S_{m}$ species transferring between phases. Also, let $p_{l}$ and $p_{g}$ be the number of inlets in each phase and let both phases have one outlet each. Assuming that (i) both phases are homogeneous, (ii) the reactions take place in the liquid bulk only, and (iii) the mass transfer phenomena can be described by the two-film theory with no accumulations in the film, the differential mole balance equations for the gas and liquid phases can be formulated as:

$$
\begin{array}{ll}
\dot{\mathbf{n}}_{g}(t)=\mathbf{W}_{\text {in }, g} \mathbf{u}_{\text {in, }, g}(t)-\mathbf{W}_{m, g} \zeta(t)-\frac{u_{\text {out }, g}(t)}{m_{g}(t)} \mathbf{n}_{g}(t), & \mathbf{n}_{g}(0)=\mathbf{n}_{g 0}(2) \\
\dot{\mathbf{n}}_{l}(t)=\mathbf{N}^{\mathrm{T}} V_{l}(t) \mathbf{r}(t)+\mathbf{W}_{\text {in }, l} \mathbf{u}_{\text {in, }, l}(t)-\mathbf{W}_{m, l} \zeta(t)-\frac{u_{\text {out }, l}(t)}{m_{l}(t)} \mathbf{n}_{l}(t), & \mathbf{n}_{l}(0)=\mathbf{n}_{l 0}(3)
\end{array}
$$

where $\mathbf{n}_{f}$ is the $S_{f}$-dimensional vector of number of moles in the $F$ phase, $f \in\{g, l\}$ and $F \in\{G, L\}, \mathbf{N}$ the $R \times S_{l}$ stoichiometric matrix, $V_{l}$ the volume of the liquid phase, $\mathbf{W}_{i n, f}=\mathbf{M}_{w, f}^{-1} \mathbf{W}_{\text {inf. }}$ the $S_{f} \times p_{f}$ inlet matrix expressing the composition of the inlets to the $F$ phase, $\mathbf{M}_{w f}$ the $S_{f}$-dimensional diagonal matrix of molecular weights,

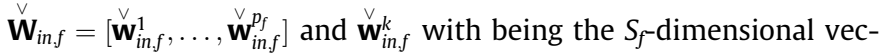
tor of weight fractions of the $k$ th inlet to the $F$ phase with $k=1, \ldots, p_{f}, \mathbf{u}_{\text {in, } f}$ the $p_{f}$-dimensional inlet mass flow rate to the $F$ phase, $u_{\text {out } f}$ the outlet mass flow rate of the $F$ phase, $m_{f}$ the mass of the $F$ phase, and $\mathbf{n}_{f 0}$ the vector of initial moles in the $F$ phase. The $p_{m}$ mass transfers are treated as pseudo inlets with unknown flow rates and involve $\mathbf{W}_{m, f}$ and $\zeta$, where $\mathbf{W}_{m, f}=\mathbf{M}_{w . f}^{-1} \mathbf{E}_{m, f}$ is the $S_{f} \times p_{m}$ mass transfer matrix for the $F$ phase, $\mathbf{e}_{m, f}=\left[\mathbf{e}_{m f}^{1}, \ldots, \breve{e}_{m . f}^{p_{m}}\right]$ with $\stackrel{\mathrm{e}}{j} j \mathrm{j}_{j}$ being the $S_{f}$-dimensional vector with the elements corresponding to the $j$ th transferring species equal to unity and the other elements equal to zero, and $\zeta$ is the $p_{m}$-dimensional vector of mass transfer rates expressed in $\frac{\text { (mass) }}{(\text { time })}$ units and defined positively $(+)$ when the mass transfer occurs from the gas to the liquid phase.

\subsection{Computation of extents from the numbers of moles}

\subsubsection{Homogeneous reaction systems}

The first step in the extent-based incremental approach is the computation of extents. Based on the mole balance equation (1), and under the condition $\operatorname{rank}\left(\left[\mathbf{N}^{\mathbf{T}} \mathbf{W}_{i n} \mathbf{n}_{0}\right]\right)=R+p+1$, Armhein et al. [17] proposed a linear transformation to decompose the $S$ dimensional vector of numbers of moles $\mathbf{n}$ into four distinct parts, namely, $R$ extents of reaction $\mathbf{x}_{r}$ (in mol units), $p$ extents of inlet flow $\mathbf{x}_{\text {in }}$ (in $\mathrm{kg}$ units), a scalar dimensionless discounting variable $\lambda$, and $(S-R-p-1)$ invariants $\mathbf{x}_{i v}$ that are identically equal to zero and thus can be discarded. The variable $\lambda$ accounts for the effect of the outlet stream on the initial conditions. This linear transformation uses only information regarding the stoichiometry $\mathbf{N}$, the inlet composition $\mathbf{W}_{i n}$ and the initial conditions $\mathbf{n}_{0}$. Applying the linear transformation to the mole balance equation (1) gives the following decoupled system:

$$
\begin{array}{ll}
\dot{\mathbf{x}}_{r}(t)=V(t) \mathbf{r}(t)-\frac{u_{\text {out }}(t)}{m(t)} \mathbf{x}_{r}(t), & \mathbf{x}_{r}(t)=\mathbf{0}_{R} \\
\dot{\mathbf{x}}_{\text {in }}(t)=\mathbf{u}_{\text {in }}(t)-\frac{u_{\text {out }}(t)}{m(t)} \mathbf{x}_{\text {in }}(t), & \mathbf{x}_{\text {in }}(0)=\mathbf{0}_{p} \\
\dot{\lambda}(t)=-\frac{u_{\text {out }}(t)}{m(t)} \lambda(t), & \lambda(0)=1
\end{array}
$$


Note that Eqs. (4) and (5) are expressed in terms of vessel extents [17], that is, the overall extents of reaction [18] are discounted for the amount of material that has left the reactor through the outlet. The vessel extents of reaction $\mathbf{x}_{r}$ are identical to the overall extents of reaction $\xi$, defined as $\dot{\xi}(t)=V(t) \mathbf{r}(t)$, for batch and semi-batch reactors because of the absence of an outlet flow. However, these two types of extents are different for continuous reactors. Vessel extents can be converted to overall extents, i.e. $\boldsymbol{\xi}(t)=\mathbf{x}_{r}(t)+\int_{0}^{t} \frac{u_{\text {out }}(\tau)}{m(\tau)} \mathbf{x}_{r}(\tau) d \tau$. The vector of number of moles $\mathbf{n}$ can be reconstructed from the extents $\mathbf{x}_{r}$, $\mathbf{x}_{\text {in }}$ and $\lambda$ as follows:

$\mathbf{n}(t)=\mathbf{N}^{\mathrm{T}} \mathbf{x}_{r}(t)+\mathbf{W}_{\text {in }} \mathbf{x}_{\text {in }}(t)+\mathbf{n}_{0} \lambda(t)$

If the inlet and outlet flow rates $\mathbf{u}_{\text {in }}$ and $\mathbf{u}_{\text {out }}$ and the mass $m$ of the reaction mixture are known, the $p$ extents of inlet flow $\mathbf{x}_{i n}$ and the discounting variable $\lambda$ can be calculated by integrating the differential equations (5) and (6), and Eq. (7) can be put in reactionvariant (RV) form [19]:

$\mathbf{n}^{\mathrm{RV}}(t):=\mathbf{n}(t)-\mathbf{W}_{i n} \mathbf{x}_{i n}(t)-\mathbf{n}_{0} \lambda(t)=\mathbf{N}^{\mathrm{T}} \mathbf{X}_{r}(t)$

from where the extents of reaction can be calculated as $\mathbf{x}_{r}(t)=\mathbf{N}^{\mathrm{T}+} \mathbf{n}^{\mathrm{RV}}(t)$ where $\mathbf{N}^{\mathrm{T}+}$ denotes the pseudo-inverse of $\mathbf{N}^{\mathrm{T}}$.

This approach can easily be adapted to compute the extents of reaction from a subset of measured species of dimension $S_{a}$, where the subscript $a$ denotes an available (measured) quantity. Eq. (8) then becomes:

$\mathbf{n}_{a}^{\mathrm{RV}}(t):=\mathbf{n}_{a}(t)-\mathbf{W}_{i n, a} \mathbf{x}_{i n}(t)-\mathbf{n}_{0, a} \lambda(t)=\mathbf{N}_{a}^{\mathrm{T}} \mathbf{x}_{r}(t)$

from which, assuming rank $\left(\mathbf{N}_{a}\right)=R$, one can write $\mathbf{x}_{r}(t)=\mathbf{N}_{a}^{\mathrm{T}+} \mathbf{n}_{a}^{\mathrm{RV}}(t)$.

\subsubsection{Gas-liquid reaction systems}

For gas-liquid reaction systems, Bhatt et al. [20] proposed to use a distinct transformation for each phase.

For the gas phase, and under the condition rank $\left(\left[\mathbf{W}_{m, g} \mathbf{W i n}_{g} \mathbf{n}_{\mathrm{g} 0}\right]\right)=p_{m}+p_{g}+1$, the $S_{g}$-dimensional vector of numbers of moles $\mathbf{n}_{g}$ is transformed into four contributions, namely, $p_{m}$ extents of mass transfer $\mathbf{x}_{m, g}$ (in $\mathrm{kg}$ units), $p_{g}$ extents of inlet flow $\mathbf{x}_{i n, g}$ (in $\mathrm{kg}$ units), a scalar dimensionless discounting variable $\lambda_{g}$, and $\left(S_{g}-p_{m}-p_{g}-1\right)$ invariants $\mathbf{x}_{i v, g}$ that are identically equal to zero and thus can be discarded. The information necessary for computing this linear transformation is $\mathbf{W}_{m, g}, \mathbf{W}_{i n, g}$ and $\mathbf{n}_{\text {go }}$.

For the liquid phase, and under the condition rank $\left(\left[\mathbf{N}^{\mathrm{T}} \mathbf{W}_{m, l} \mathbf{W}_{i n, l} \mathbf{n}_{l 0}\right]\right)=R+p_{m}+p_{l}+1$, the $S_{l}$-dimensional vector of numbers of moles $\mathbf{n}_{l}$ is transformed into five contributions, namely, $R$ extents of reactions $\mathbf{x}_{r}$ (in mol units), $p_{m}$ extents of mass transfer $\mathbf{x}_{m, l}$ (in kg units), $p_{l}$ extents of inlet flow $\mathbf{x}_{i n, l}$ (in $\mathrm{kg}$ units), a scalar dimensionless discounting variable $\lambda_{l}$, and $\left(S_{l}-R-p_{m}-p_{l}-1\right)$ invariants $\mathbf{x}_{i v, l}$ that are identically equal to zero and thus can be discarded. This linear transformation uses only information about $\mathbf{N}$, $\mathbf{W}_{m, l}, \mathbf{W}_{i n, l}$ and $\mathbf{n}_{l 0}$.

Applying the linear transformations to the mole balance equations (2) and (3) gives, for each phase $f \in\{g, l\}$, the following decoupled system:

$$
\begin{array}{ll}
\dot{\mathbf{x}}_{r}(t)=V_{l}(t) \mathbf{r}(t)-\frac{u_{\text {out }, l}(t)}{m_{l}(t)} \mathbf{x}_{r}(t), & \mathbf{x}_{r}(t)=\mathbf{0}_{R} \\
\dot{\mathbf{x}}_{m, f}(t)=\zeta(t)-\frac{u_{\text {out } f f}(t)}{m_{f}(t)} \mathbf{x}_{m, f}(t), & \mathbf{x}_{m, f}(0)=\mathbf{0}_{p_{m}} \\
\dot{\mathbf{x}}_{\text {inf } f}(t)=\mathbf{u}_{\text {inf } f}(t)-\frac{u_{\text {out } f}(t)}{m_{f}(t)} \mathbf{x}_{\text {inf } f}(t), & \mathbf{x}_{\text {inf } f}(0)=\mathbf{0}_{p_{f}} \\
\dot{\lambda}_{f}(t)=-\frac{u_{\text {out } f f}(t)}{m_{f}(t)} \lambda_{f}(t), & \lambda_{f}(0)=1
\end{array}
$$

Note that, as the reactions are assumed to take place in the liquid bulk only, Eq. (10) applies only to the liquid phase. The vectors of numbers of moles can be reconstructed from the extents as follows:

$\mathbf{n}_{g}(t)=-\mathbf{W}_{m, g} \mathbf{x}_{m, g}(t)+\mathbf{W}_{i n, g} \mathbf{x}_{i n, g}(t)+\mathbf{n}_{g 0} \lambda_{g}(t)$

$\mathbf{n}_{l}(t)=\mathbf{N}^{\mathrm{T}} \mathbf{x}_{r}(t)+\mathbf{W}_{m, l} \mathbf{x}_{m, l}(t)+\mathbf{W}_{i n, l} \mathbf{x}_{i n, l}(t)+\mathbf{n}_{l 0} \lambda_{l}(t)$

As in the case of homogeneous reaction systems, if the inlet and outlet flow rates $\mathbf{u}_{\text {inf } f}$ and $u_{\text {out } f}$ in each phase and the mass $m_{f}$ of each reaction mixture are known, the $p_{f}$ extents of inlet flow $\mathbf{x}_{i n f}$ and the discounting variables $\lambda_{f}$ can be calculated by integration of the differential equations (12) and (13). Bhatt et al. [20] proposed to compute the mass-transfer-variant (MV), reaction-variant (RV), and reaction- and mass-transfer-variant (RMV) quantities in three steps:

1. Rearrange Eq. (14) in MV-form and calculate the $p_{m}$ extents of mass transfer $\mathbf{x}_{m, g}$ in the gas phase using the pseudo-inverse of $\mathbf{W}_{m, g}$ :

$$
\mathbf{n}_{g}^{\mathrm{MV}}(t):=\mathbf{n}_{g}(t)-\mathbf{W}_{i n, g} \mathbf{x}_{i n, g}(t)-\mathbf{n}_{g 0} \lambda_{g}(t)=-\mathbf{W}_{m, g} \mathbf{x}_{m, g}(t)
$$

2. Convert the extents of mass transfer in the gas phase to extents of mass transfer in the liquid phase using $\mathbf{x}_{m, l}=\mathbf{x}_{m, g}-\boldsymbol{\delta}_{m}$, with the auxiliary variables $\boldsymbol{\delta}_{m}$ obtained by integration of the following differential equations:

$$
\begin{aligned}
& \dot{\boldsymbol{\delta}}_{m}(t)=-\frac{u_{\text {out }, l}(t)}{m_{l}(t)} \boldsymbol{\delta}_{m}(t)+\left(\frac{u_{\text {out }, l}(t)}{m_{l}(t)}-\frac{u_{\text {out }, g}(t)}{m_{g}(t)}\right) \mathbf{x}_{m, g}(t), \\
& \boldsymbol{\delta}_{m}(0)=\mathbf{0}_{p_{m}}
\end{aligned}
$$

3. Rearrange Eq. (15) in RV-form and use the extents $\mathbf{x}_{m, l}$ to compute the $R$ extents of reaction $\mathbf{x}_{r}$ by means of the pseudo-inverse of $\mathbf{N}^{\mathrm{T}}$ :

$$
\mathbf{n}_{l}^{\mathrm{RV}}(t):=\mathbf{n}_{l}(t)-\mathbf{W}_{m, l} \mathbf{x}_{m, l}(t)-\mathbf{W}_{i n, l} \mathbf{x}_{i n, l}(t)-\mathbf{n}_{l 0} \lambda_{l}(t)=\mathbf{N}^{\mathrm{T}} \mathbf{x}_{r}(t)
$$

This procedure can also be adapted to compute the extents of reaction and mass transfer for a subset of $S_{g, a}$ and $S_{l, a}$ number of moles measurements. Let $p_{m_{f}}$ be the number of mass transfers that are computed from the $F$ phase, with $p_{m_{g}}+p_{m_{l}}=p_{m}$. The procedure is then the following:

1. Rearrange Eq. (14) in MV-form and calculate the $p_{m_{g}}$ extents of mass transfer $\mathbf{x}_{m_{g} . g}$ in the gas phase using the pseudo-inverse of $\mathbf{W}_{m_{g}, g, a}$ :

$$
\mathbf{n}_{g, a}^{\mathrm{MV}}(t):=\mathbf{n}_{g, a}(t)-\mathbf{W}_{i n, g, a} \mathbf{x}_{i n, g}(t)-\mathbf{n}_{g 0, a} \lambda_{g}(t)=-\mathbf{W}_{m_{g}, g, a} \mathbf{x}_{m_{g}, g}(t)
$$

2. Convert the $p_{m_{g}}$ extents of mass transfer calculated from the gas phase to extents of mass transfer in the liquid phase using $\mathbf{x}_{m_{g}, l}=\mathbf{x}_{m_{g} g}-\boldsymbol{\delta}_{m_{g}}$, with the auxiliary variables $\boldsymbol{\delta}_{m_{g}}$ obtained by integration of the following differential equations:

$$
\dot{\boldsymbol{\delta}}_{m_{g}}(t)=-\frac{u_{\text {out }, l}(t)}{m_{l}(t)} \boldsymbol{\delta}_{m_{g}}(t)+\left(\frac{u_{\text {out }, l}(t)}{m_{l}(t)}-\frac{u_{\text {out }, g}(t)}{m_{g}(t)}\right) \mathbf{x}_{m_{g} g}(t) \boldsymbol{\delta}_{m_{g}}(0)=\mathbf{0}_{p_{m g}}
$$

3. Rearrange Eq. (15) in RMV-form and use the extents $\mathbf{x}_{m_{g}, l}$ to compute the $R$ extents of reaction $\mathbf{x}_{r}$ and the $p_{m_{l}}=p_{m}-p_{m_{g}}$ remaining extents of mass transfer $\mathbf{x}_{m_{l}, l}$ by means of the pseudo-inverse of the matrix $\left[\mathbf{N}_{a}^{\mathrm{T}} \mathbf{W}_{m_{l}, l, a}\right]$ : 


$$
\begin{aligned}
\mathbf{n}_{l, a}^{\mathrm{RMV}}(t) & :=\mathbf{n}_{l, a}(t)-\mathbf{W}_{m_{g}, l, a} \mathbf{x}_{m_{g}, l}(t)-\mathbf{W}_{i n, l, a} \mathbf{x}_{i n, l}(t)-\mathbf{n}_{l 0, a} \lambda_{l}(t) \\
& =\left[\mathbf{N}_{a}^{\mathrm{T}} \mathbf{W}_{m_{l}, l, a}\right]\left[\begin{array}{c}
\mathbf{x}_{r}(t) \\
\mathbf{x}_{m_{l}, l}(t)
\end{array}\right]
\end{aligned}
$$

This procedure is based on the two conditions, $S_{g, a}+S_{l, a} \geqslant R+p_{m}$ and $\operatorname{rank}\left(\left[\mathbf{N}_{a}^{\mathrm{T}} \mathbf{W}_{m_{l}, l, a}\right]\right)=R+p_{m_{l}}$.

\subsection{Model identification based on computed extents}

In the second step of the extent-based incremental approach, the rate laws and the corresponding parameters are identified using the extents of reaction and mass transfer computed in the previous step. A set of rate law candidates is postulated, and a least-squares regression problem involving a dynamic model as constraint is formulated for each extent of reaction and each mass transfer. Individual rate laws are integrated and the corresponding parameters estimated by comparing predicted and computed extents.

For kinetic identification, the regression problem is constrained by Eq. (4) for homogeneous reaction systems and Eq. (10) for gas-liquid reaction systems. The $i$ th dynamic model contains a postulated rate law of the form $r_{i}\left(\mathbf{c}(t), \boldsymbol{\theta}_{r, i}\right)$ with $\mathbf{c}(t)=\frac{\mathbf{n}(t)}{V(t)}$, for homogeneous reaction systems and $r_{i}\left(\mathbf{c}_{l}(t), \boldsymbol{\theta}_{r, i}\right)$, with $\mathbf{c}_{l}(t)=\frac{\mathbf{n}_{l}(t)}{V_{l}(t)}$ for gas-liquid reaction systems. Each postulated rate law depends on a set of adjustable parameters $\boldsymbol{\theta}_{r, i}$, which are typically rate constants and activation energies. For mass-transfer rate identification, the regression problem is constrained by Eq. (11). The $j$ th dynamic model involves a postulated rate law of the form $\zeta_{j}\left(\mathbf{c}_{l}(t), \mathbf{c}_{\mathrm{g}}(t), \boldsymbol{\theta}_{m_{j}}\right)$, with $\mathbf{c}_{g}(t)=\frac{\mathbf{n}_{g}(t)}{V_{g}(t)}$, which depends on the adjustable parameters $\boldsymbol{\theta}_{m, j}$ and on known properties of the system, such as Henry's constants and Raoult's vapor pressures. Mass-transfer coefficients are examples of adjustable parameters for mass-transfer rate laws.

\subsection{Calorimetric data for rank augmentation}

\subsubsection{Homogeneous reaction systems}

Calorimetric data can be measured as a differential (heat flow) signal in [W] or as an integral heat signal in [J]. Differential and integral calorimetric signals are generally obtained by energy balances and appropriate measurements of temperature and flow rates. For homogeneous reaction systems, the heat flow signal $q_{r}$ is defined as:

$q_{r}(t)=\dot{\mathbf{x}}_{r}^{\mathrm{T}}(t)\left(-\Delta \mathbf{H}_{r}\right)$

where $\dot{\mathbf{x}}_{r}$ is given by Eq. (4) and $\Delta \mathbf{H}_{r}$ is the $R$-dimensional vector of reaction enthalpies in $\left[\mathrm{J} \mathrm{mol}^{-1}\right]$. Note that the negative sign stems from the convention that exothermic reactions have negative reaction enthalpies. As the heat flow is defined in terms of vessel extents, $q_{r}(t)$ can be interpreted as the reaction heat flow that is still present in the reactor at time $t$. Based on this definition of $q_{r}$, the integral heat signal $Q_{r}$ is defined as:

$Q_{r}(t)=\int_{0}^{t} q_{r}(\tau) d \tau=\mathbf{x}_{r}^{\mathrm{T}}(t)\left(-\Delta \mathbf{H}_{r}\right)$

For rank $\left(\mathbf{N}_{a}\right)=R-1$, that is, when the number of measured species $S_{a}$ is insufficient to compute the $R$ extents of reaction, the heat signal $Q_{r}(t)$ can be used as an additional measured signal to augment by one the number of measured quantities, thereby allowing the computation of the $R$ extents of reaction. Eq. (9) can be modified as follows: $\mathbf{n}_{a u g}^{\mathrm{RV}}(t):=\left[\begin{array}{c}\mathbf{n}_{a}^{\mathrm{RV}}(t) \\ Q_{r}(t)\end{array}\right]=\left[\begin{array}{c}\mathbf{N}_{a}^{\mathrm{T}} \\ -\Delta \mathbf{H}_{r}^{\mathrm{T}}\end{array}\right] \mathbf{x}_{r}(t):=\mathbf{N}_{a u g}^{\mathrm{T}} \mathbf{x}_{r}(t)$

with $\mathbf{n}_{\text {aug }}^{\mathrm{RV}}$ the augmented vector of measurements in RV form of dimension $\left(S_{a}+1\right)$ and $\mathbf{N}_{a u g}$ an augmented $\left[R \times\left(S_{a}+1\right)\right]$-dimensional matrix of rank $R$. Since the augmented matrix $\mathbf{N}_{a u g}$ is of rank $R$, the $R$ extents of reaction $\mathbf{x}_{r}$ can be obtained from $\mathbf{n}_{\text {aug }}^{\mathrm{RV}}$ and using the pseudo-inverse of $\mathbf{N}_{\text {aug }}^{\mathrm{T}}$. Note that this rank augmentation is only possible if all reaction enthalpies $\Delta \mathbf{H}_{r}$ are known.

\subsubsection{Gas-liquid reaction systems}

The relationship between the calorimetric signal $q_{r}$ and the extents of reaction $\mathbf{x}_{r}$ given in Eq. (22) also holds for gas-liquid reaction systems under the condition that either the transferring species involved in the liquid-phase reactions are specified in gaseous form or the heat effects due to mass transfer are negligible. In such a case, Eq. (22) can be used with $\dot{\mathbf{x}}_{r}$ given by Eqs. (10) and (23) can also be used for the definition of the integral heat signal $Q_{r}$.

More generally, if none of the two aforementioned conditions are met, the heat effect due to mass transfer has to be explicitly taken into account, with the heat flow $q_{r m}$ due to reaction and mass transfer defined as follows:

$$
q_{r m}(t)=\dot{\mathbf{x}}_{r}^{\mathrm{T}}(t)\left(-\Delta \mathbf{H}_{r}\right)+\dot{\mathbf{x}}_{m, l}^{\mathrm{T}}(t)\left(-\Delta \mathbf{H}_{m}\right)=\left[\begin{array}{ll}
\dot{\mathbf{x}}_{r}^{\mathrm{T}}(t) & \dot{\mathbf{x}}_{m, l}^{\mathrm{T}}(t)
\end{array}\right]\left[\begin{array}{c}
-\Delta \mathbf{H}_{r} \\
-\Delta \mathbf{H}_{m}
\end{array}\right]
$$

where $\dot{\mathbf{x}}_{m, l}$ is given by Eq. (11) and expressed in [kg] and $\Delta \mathbf{H}_{m}$ the $p_{m}$-dimensional vector of enthalpies of mass transfer expressed in $\left[\mathrm{J} \mathrm{kg}^{-1}\right]$. It follows that the integral heat $Q_{r m}$ becomes:

$Q_{r m}(t)=\int_{0}^{t} q_{r m}(\tau) d \tau=\left[\begin{array}{ll}\mathbf{x}_{r}^{\mathrm{T}}(t) & \mathbf{x}_{m, l}^{\mathrm{T}}(t)\end{array}\right]\left[\begin{array}{l}-\Delta \mathbf{H}_{r} \\ -\Delta \mathbf{H}_{m}\end{array}\right]$

For rank $\left(\left[\mathbf{N}_{a}^{\mathrm{T}} \mathbf{W}_{m_{l}, l, a}\right]\right)=R+p_{m_{l}}-1$, that is, when the number of measured species $S_{l, a}$ is insufficient to compute the $R$ extents of reaction and the $p_{m_{l}}$ mass transfers, the heat signal $Q_{r m}$ can be used as an additional measured quantity, thereby allowing the computation of $\mathbf{x}_{r}$ and $\mathbf{x}_{m, l .}$ Eq. (21) can be modified as follows:

$$
\begin{aligned}
\mathbf{n}_{l, a u g}^{\mathrm{RMV}}(t) & :=\left[\begin{array}{c}
\mathbf{n}_{l, a}^{\mathrm{RMV}}(t) \\
Q_{r m}(t)-Q_{m_{g}, l}(t)
\end{array}\right]=\left[\begin{array}{cc}
\mathbf{N}_{a}^{\mathrm{T}} & \mathbf{W}_{m_{l}, l, a} \\
-\Delta \mathbf{H}_{r}^{\mathrm{T}} & -\Delta \mathbf{H}_{m_{l}}^{\mathrm{T}}
\end{array}\right]\left[\begin{array}{c}
\mathbf{x}_{r}(t) \\
\mathbf{x}_{m_{l}, l}(t)
\end{array}\right] \\
& :=\mathbf{N}_{l, a u g}^{\mathrm{T}}\left[\begin{array}{c}
\mathbf{x}_{r}(t) \\
\mathbf{x}_{m_{l}, l}(t)
\end{array}\right]
\end{aligned}
$$

with $Q_{m_{g}, l}(t)=\mathbf{x}_{m_{g}, l}^{\mathrm{T}}(t)\left(-\Delta \mathbf{H}_{m_{g}}\right)$

where $\mathbf{n}_{l, \text { aug }}^{\mathrm{RMV}}$ is the augmented $\left(S_{l, a}+1\right)$-dimensional vector of numbers of moles in RMV form, $\Delta \mathbf{H}_{m_{g}}$ the $p_{m_{g}}$-dimensional vector of enthalpies of mass transfer corresponding to the $p_{m_{g}}$ extents of mass transfer $\mathbf{x}_{m_{\mathrm{g}, l},}, \Delta \mathbf{H}_{m_{l}}$ the $p_{m_{l}}$-dimensional vector of enthalpies of mass transfer associated to the $p_{m_{l}}$ extents of mass transfer $\mathbf{x}_{m_{l}, l}$, and $\mathbf{N}_{l, \text { aug }}$ an augmented $\left[\left(R+p_{m_{l}}\right) \times\left(S_{l, a}+1\right)\right]$-dimensional matrix of rank $R+p_{m_{l}}$. Note that the term $Q_{m_{g}, l}$ that is subtracted from the heat signal $Q_{r m}$ is necessary to remove the heat effect of the $p_{m_{g}}$ mass transfers that are already calculated from the gas phase. As the $\left(S_{l, a}+1\right)$ measured data are now of rank $R+p_{m_{l}}$, the $R$ extents of reaction and the $p_{m_{l}}$ remaining extents of mass transfer can be obtained from $\mathbf{n}_{l, a u g}^{\mathrm{RMV}}$ using the pseudo-inverse of matrix $\mathbf{N}_{l, a u g}^{\mathrm{T}}$. Note that the rank augmentation from $\mathbf{N}_{a}$ to $\mathbf{N}_{l, a u g}$ is only possible if the enthalpies $\Delta \mathbf{H}_{r}$ and $\Delta \mathbf{H}_{m}$ are known.

Eq. (27) can be simplified when all $S_{g}$ mole profiles are measured in the gas phase and consequently when all the $p_{m}$ extents of mass transfer are computed from the gas phase. In such a case, no additional extents of mass transfer have to be calculated from the liquid phase and the vector of numbers of moles in the liquid phase $\mathbf{n}_{l, a}$ can be rearranged in RV form to compute the $R$ extents of reaction. If $\operatorname{rank}\left(\mathbf{N}_{a}\right)=R-1, Q_{r m}$ can serve as an additional signal 
in order to augment by one the number of measured quantities. The resulting equation is similar to Eq. (24) with $Q_{r}(t)=Q_{r m}(t)-Q_{m}(t)$ and $Q_{m}(t)=\mathbf{x}_{m, l}^{\mathrm{T}}(t)\left(-\Delta \mathbf{H}_{m}\right)$.

\subsection{Calorimetric data for estimating enthalpies}

\subsubsection{Homogeneous reaction systems}

When the extents of reaction can be directly calculated by means of either the linear transformation or the RV form of the number of moles profiles, that is, without any rank augmentation using calorimetric data, these extents can be used subsequently to estimate the reaction enthalpies $\Delta \mathbf{H}_{r}$ from the measured heat signal $Q_{r}$. This can be done by left-multiplying Eq. (23) with the pseudo-inverse of $\mathbf{x}_{r}^{\mathrm{T}}$ :

$\left(-\Delta \mathbf{H}_{r}\right)=\mathbf{x}_{r}^{\mathrm{T}+}(t) Q_{r}(t)$

\subsubsection{Gas-liquid reaction systems}

Similarly, for gas-liquid reaction systems, if the integral heat signal $Q_{r}$ has not been used as an additional measurement to compute the various extents, $Q_{r m}$ allows estimating the enthalpies of reaction and mass transfer from the computed extents. Indeed, left-multiplying Eq. (26) with the pseudo-inverse of $\left[\mathbf{x}_{r}^{\mathrm{T}}(t) \mathbf{x}_{m, l}^{\mathrm{T}}(t)\right]$ gives:

$\left[\begin{array}{c}-\Delta \mathbf{H}_{r} \\ -\Delta \mathbf{H}_{m}\end{array}\right]=\left[\mathbf{x}_{r}^{\mathrm{T}}(t) \mathbf{x}_{m, l}^{\mathrm{T}}(t)\right]^{+} Q_{r m}(t)$

\section{Case studies}

Two simulation studies are carried out in this section. The first investigation, which considers the homogeneous acetoacetylation of pyrrole in an isothermal semi-batch reactor, illustrates the use of calorimetry to increase the rank of the measurement equations. In the second investigation dealing with the gas-liquid chlorination of butanoic acid in a semi-batch reactor, calorimetric data are used to estimate reaction enthalpies.

\subsection{Rank augmentation - Homogeneous acetoacetylation of pyrrole}

The acetoacetylation of pyrrole (A) with diketene (B) involves one main reaction and three side reactions. The main reaction $\mathrm{R} 1$ between pyrrole and diketene produces the desired 2-acetoacetyl pyrrole (C). The side reactions include the dimerization $\mathrm{R} 2$ of diketene to dehydroacetic acid (D), the oligomerization R3 of diketene to oligomers (E) and the reaction R4 of diketene and acetoacetyl pyrrole giving by-product (F). Reactions R1, R2 and R4 are all catalyzed by pyridine (K). These four homogeneous reactions can be represented by the following stoichiometric and kinetic expressions:

$$
\begin{array}{lrl}
\mathrm{R} 1: & \mathrm{A}+\mathrm{B} \rightarrow \mathrm{C} & r_{1}=k_{1} c_{A} c_{B} c_{K} \\
\mathrm{R} 2: & \mathrm{B}+\mathrm{B} \rightarrow \mathrm{D} & r_{2}=k_{2} c_{B}^{2} c_{K} \\
\mathrm{R} 3: & \mathrm{B} \rightarrow \mathrm{E} & r_{3}=k_{3} c_{B} \\
\mathrm{R} 4: & \mathrm{B}+\mathrm{C} \rightarrow \mathrm{F} & r_{4}=k_{4} c_{C} c_{B} c_{K}
\end{array}
$$

For simulating the reaction system, the values of the rate constants $k_{1}-k_{4}$ are evaluated using Arrhenius equation. The values of the activation energies, frequency factors and reaction enthalpies used in the simulation are taken from [21]. The reaction is conducted in an isothermal semi-batch reactor at $323 \mathrm{~K}$. The initial concentrations of the $S=6$ species are $\mathbf{c}_{0}=\left[\begin{array}{llllll}0.72 & 0.09 & 0.10 & 0.02 & 0 & 0\end{array}\right]^{\mathrm{T}} \mathrm{mol} \mathrm{L}^{-1}$, and $5 \mathrm{~mol}$ of catalyst $(\mathrm{K})$ are initially present in the reactor. The initial volume of the reaction mixture is $1 \mathrm{~L}$. Species B is added continuously with the constant volumetric flow rate $q_{\text {in }}=0.009 \mathrm{~L} \mathrm{~min}^{-1}$ and the composition $\mathbf{c}_{i n}=\left[\begin{array}{llllll}0 & 5.82 & 0 & 0 & 0 & 0\end{array}\right]^{\mathrm{T}} \mathrm{mol} \mathrm{L}^{-1}$. The density of the reaction mixture is assumed to be constant throughout the course of the reaction. The concentrations $\mathbf{c}_{a}(t)=\frac{\mathbf{n}_{a}(t)}{V(t)}$ of $S_{a}=3$ species $\{A, B, C\}$ are assumed to be measured every minute for $145 \mathrm{~min}$. Each concentration measurement is corrupted by additive zeromean Gaussian noise with standard deviation corresponding to $1 \%$ of that species maximal concentration. For the subset of measured species, the stoichiometry matrix $\mathbf{N}_{a}$ of dimension $(4 \times 3)$ is given by:

$\mathbf{N}_{a}=\left[\begin{array}{ccc}-1 & -1 & 1 \\ 0 & -2 & 0 \\ 0 & -1 & 0 \\ 0 & -1 & -1\end{array}\right]$

As the rank of $\mathbf{N}_{a}$ is 3 , neither the linear transformation nor the $\mathrm{RV}$ form of the concentration profiles can be used to compute the 4 extents of reaction. It is necessary to measure one additional species or to use calorimetry to be able to compute all extents of reactions. For example, using the integral heat signal $Q_{r}$, the observed signal $\mathbf{n}_{\text {aug }}^{\mathrm{RV}}$ is of dimension 4 and the augmented matrix $\mathbf{N}_{\text {aug }}$ of rank 4 as described in Eq. (24), thereby allowing the computation of $\mathbf{x}_{r}$. The heat flow signal $q_{r}$ is simulated using Eq. (22) with $u_{\text {out }}=0$ (semi-batch reactor); zero-mean Gaussian noise with standard deviation corresponding to $3 \%$ of the maximum heat flow signal is added to $q_{r}$. The enthalpies of reaction that are used for generating the calorimetric data are given in Table 1 . The concentration measurements $\mathbf{c}_{a}(t)$ and the calorimetric signal $q_{r}(t)$ are shown in Fig. 1.

The heat flow $q_{r}$ is integrated to calculate the heat signal $Q_{r}$, which is subsequently used in Eq. (24) to augment $\mathbf{n}_{a}^{\mathrm{RV}}$ to $\mathbf{n}_{a u g}^{\mathrm{RV}}$. The four extents of reaction $\mathbf{x}_{r}$ are then computed using the pseudo-inverse of the augmented matrix $\mathbf{N}_{a u g}^{\mathrm{T}}$. Finally, the $i$ th rate law $r_{i}$ and the rate constant $\hat{k}_{i}$ are identified individually by minimizing in the least-squares sense the difference between the $i$ th computed extent $x_{r, i}$ and the corresponding predicted extent $\hat{x}_{r, i}$ obtained by integration of the rate law $r_{i}$ with the rate constant $\hat{k}_{i}$. Fig. 2a shows the extent of the first reaction computed from measurements, $x_{r, 1}$ along with the extent predicted by various models, $\hat{x}_{r, 1}$ and their

Table 1

\begin{tabular}{|c|c|c|c|c|c|c|}
\hline \multirow[t]{2}{*}{ Reaction } & \multicolumn{2}{|l|}{ Simulation } & \multicolumn{2}{|c|}{ Estimation (1\% and 3\% noise) } & \multicolumn{2}{|c|}{ Estimation ( $5 \%$ and $5 \%$ noise) } \\
\hline & $\Delta H_{r, i}$ & $k_{i}$ & $\hat{k}_{i}$ & $99 \%$ C.I. & $\hat{k}_{i}$ & $99 \%$ C.I. \\
\hline R1 & -132.69 & 0.0530 & 0.0531 & {$[0.0529,0.0532]$} & 0.0529 & {$[0.0522,0.0536]$} \\
\hline $\mathrm{R} 2$ & -91.92 & 0.1280 & 0.1279 & {$[0.1277,0.1281]$} & 0.1283 & {$[0.1275,0.1290]$} \\
\hline R3 & -1426.12 & 0.0280 & 0.0281 & {$[0.0280,0.0281]$} & 0.0278 & {$[0.0277,0.0280]$} \\
\hline R4 & -132.69 & 0.0030 & 0.0030 & {$[0.0029,0.0031]$} & 0.0030 & {$[0.0025,0.0034]$} \\
\hline
\end{tabular}

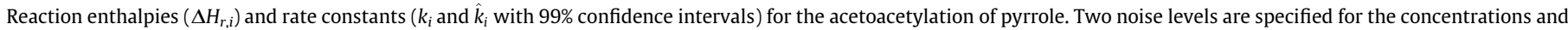

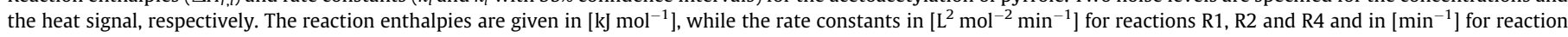
R3. 
(a)

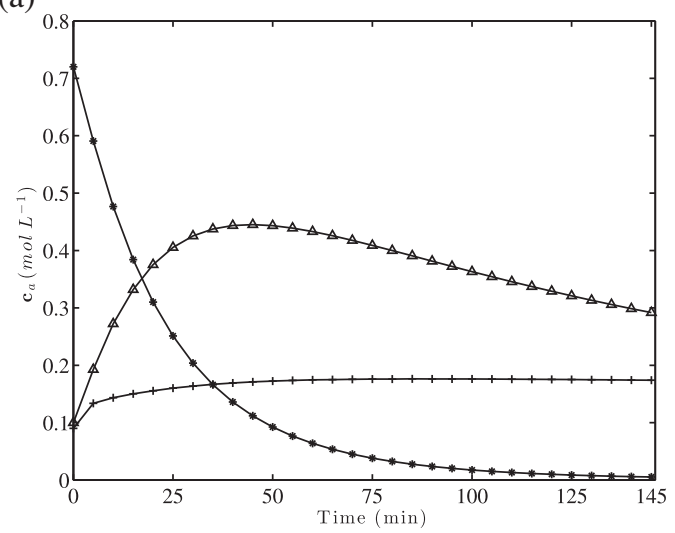

(b)

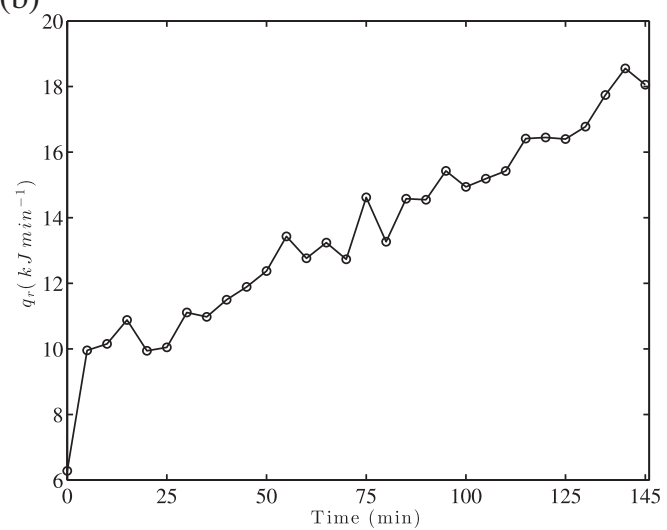

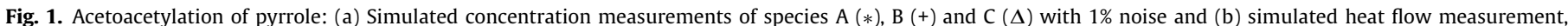
measured every minute but plotted every 5 minutes, with $3 \%$ noise.
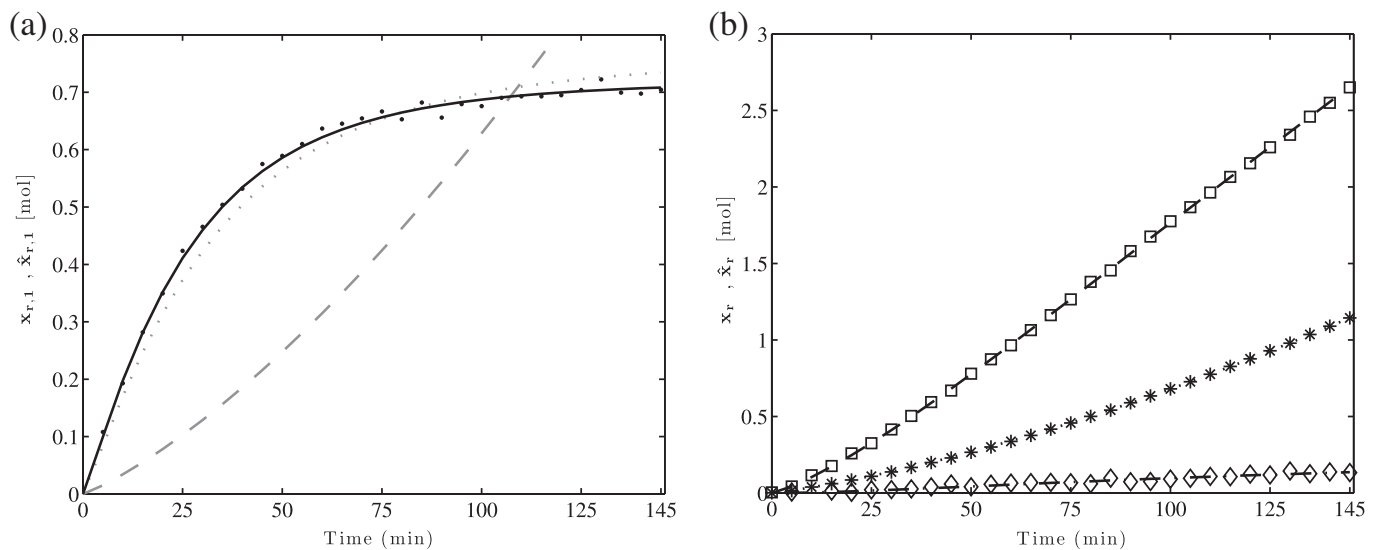

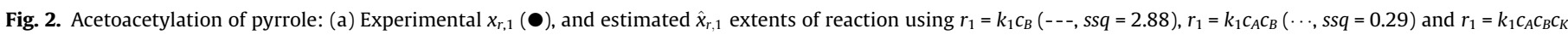
$(-, s s q=0.09)$ and (b) experimental $x_{r, 2}(\square), x_{r, 3}(*), x_{r, 4}(\diamond)$, and estimated $\hat{x}_{r, 2}(---), \hat{x}_{r, 3}(---), \hat{x}_{r, 4}(-.-)$ extents of reaction, plotted every 5 minutes.

corresponding sum of squares. It shows that the best model in the least-squares sense coincides with the model used for simulation. The same procedure was adopted for the identification of the model structure of the three other reactions (not shown). The computed and estimated extents of reaction for R2, R3 and R4 are shown in Fig. 2b. The estimated rate constants for all reactions are given in Table 1. To test the effect of noise on the estimated kinetic parameters, both the concentrations and the calorimetric signals were corrupted with 5\% zero-mean Gaussian noise. The estimated parameter values obtained with higher noise levels are essentially the same, as shown in Table 1.

\subsection{Estimation of enthalpies - Gas-liquid chlorination of butanoic acid}

The chlorination of butanoic acid (BA) is a gas-liquid reaction system involving two reactions in the liquid phase that consume chlorine $\left(\mathrm{Cl}_{2}\right)$ dissolved from the gas phase. The first reaction produces $\alpha$-mono-chloro-butanoic acid (MBA) and hydrochloric acid $(\mathrm{HCl})$, while the second reaction produces the side product $\alpha$ di-chloro-butanoic acid (DBA) and $\mathrm{HCl}$. Due to its high volatility, $\mathrm{HCl}$ can be found in both phases. Hence, this reaction system has two species in the gas phase $\left\{\mathrm{Cl}_{2}, \mathrm{HCl}\right\}$ and five species in the liquid phase $\left\{\mathrm{Cl}_{2}, \mathrm{BA}, \mathrm{MBA}, \mathrm{HCl}, \mathrm{DBA}\right\}$. Ethanol is generally used as solvent for the liquid phase. The reaction can be represented by the following scheme:

$$
\begin{aligned}
& \mathrm{R} 1: \mathrm{BA}_{(l)}+\mathrm{Cl}_{2(g)} \rightarrow \mathrm{MBA}_{(l)}+\mathrm{HCl}_{(g)} \\
& \mathrm{R} 2: \mathrm{BA}_{(l)}+2 \mathrm{Cl}_{2(g)} \rightarrow \mathrm{DBA}_{(l)}+2 \mathrm{HCl}_{(g)}
\end{aligned}
$$

The rate laws for reactions $\mathrm{R} 1$ and $\mathrm{R} 2$ and the mass transfer rates of $\mathrm{Cl}_{2}$ and $\mathrm{HCl}$ are:

$r_{1}=\frac{k_{1} \sqrt{c_{M B A}+k_{2}}}{1+k_{3} c_{\mathrm{Cl}_{2}}}\left(\frac{c_{B A}}{c_{B A}+\varepsilon_{1}}\right)\left(\frac{c_{\mathrm{Cl}_{2}}}{c_{\mathrm{Cl}_{2}}+\varepsilon_{2}}\right)$

$r_{2}=k_{4} r_{1} c_{\mathrm{Cl}_{2}}$

$\zeta_{\mathrm{Cl}_{2}}=k_{\mathrm{Cl}_{2}} A V_{l} M_{w, \mathrm{Cl}_{2}}\left(c_{\mathrm{Cl}_{2}}^{*}-c_{\mathrm{Cl}_{2}}\right)$

$\zeta_{\mathrm{HCl}}=k_{\mathrm{HCl}} A V_{l} M_{w, \mathrm{HCl}}\left(c_{\mathrm{HCl}}-c_{\mathrm{HCl}}^{*}\right)$

where $k_{1}, k_{2}, k_{3}$ and $k_{4}$ are rate constants, $c_{\mathrm{Cl}_{2}}^{*}$ and $c_{\mathrm{HCl}}^{*}$ are the equilibrium concentrations of $\mathrm{Cl}_{2}$ and $\mathrm{HCl}$ at the interface, calculated

Table 2

Kinetic parameters and mass transfer coefficients used for simulation of chlorination of butanoic acid system.

\begin{tabular}{llll}
\hline Parameter & Value & Parameter & Value \\
\hline$k_{1}\left(\left(\mathrm{kmol} \mathrm{m}^{-3}\right)^{1 / 2} \mathrm{~s}^{-1}\right)$ & 0.0044 & $k_{\mathrm{Cl}_{2}}\left(\mathrm{~m} \mathrm{~s}^{-1}\right)$ & $0.666 \times 10^{-4}$ \\
$k_{2}\left(\mathrm{kmol} \mathrm{m}^{-3} \mathrm{~s}^{-1}\right)$ & 0.0088 & $k_{\mathrm{HCl}}\left(\mathrm{m} \mathrm{s}^{-1}\right)$ & $0.845 \times 10^{-4}$ \\
$k_{3}\left(\mathrm{~m}^{3} \mathrm{kmol}^{-1}\right)$ & 1.3577 & $A\left(\mathrm{~m}^{-1}\right)$ & 254.9 \\
$k_{4}$ & 0.1 & $\varepsilon_{1}$ & 0.2 \\
& & $\varepsilon_{2}$ & 0.001 \\
\hline
\end{tabular}


(a)

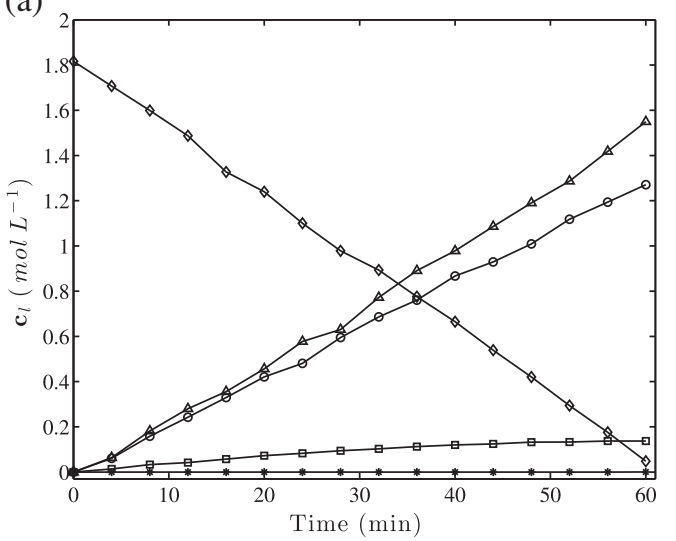

(b)

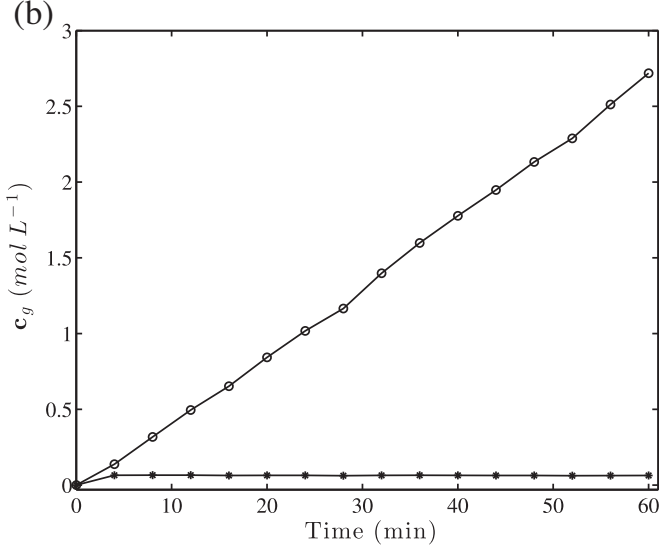

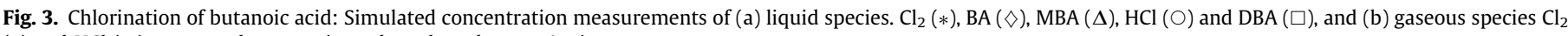
$(*)$ and $\mathrm{HCl}(\bigcirc)$, measured every minute but plotted every 4 minutes.

(a)

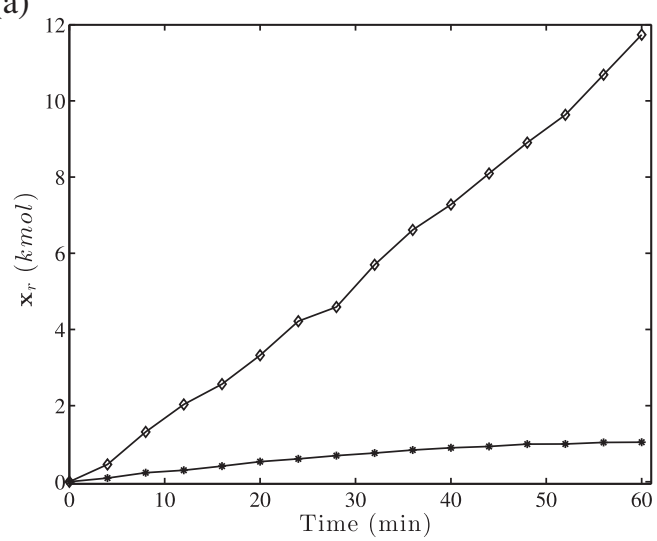

(b)

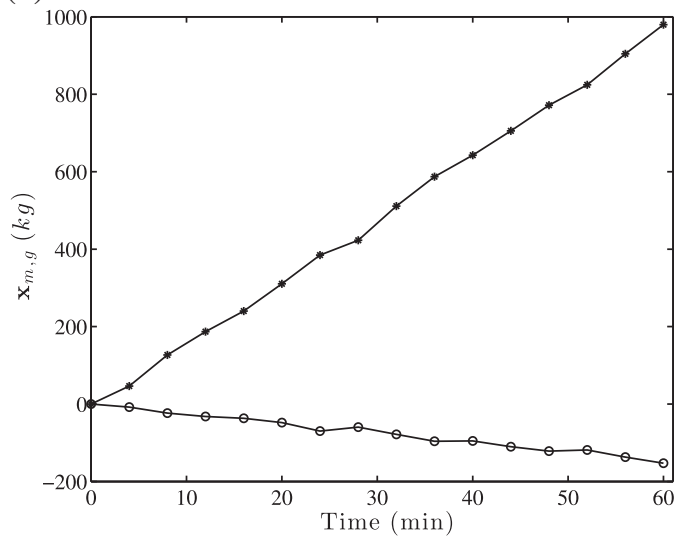

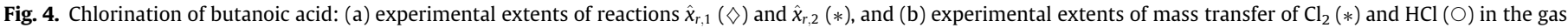
phase, plotted every 4 minutes.

using Henry constants, $k_{\mathrm{Cl}_{2}}$ and $k_{\mathrm{HCl}}$ are the molar transfer coefficients of $\mathrm{Cl}_{2}$ and $\mathrm{HCl}$ and $A$ is the specific interfacial area. The values of the reaction parameters and coefficients are taken from [20] and are given in Table 2 .

For the simulation, $13 \mathrm{kmol}$ of butanoic acid, a small amount of MBA and $100 \mathrm{kmol}$ of solvent are initially loaded in the reactor. The reactor is operated in semi-batch mode and continuously fed with chlorine gas with the mass flow rate $u_{i n, g}=972 \mathrm{~kg} \mathrm{~h}^{-1}$. The reactor volume $V_{r}$ is constant at $9 \mathrm{~m}^{3}$, with the gas and liquid phases initially occupying $1.844 \mathrm{~m}^{3}$ and $7.156 \mathrm{~m}^{3}$, respectively. The concentrations in the gas and liquid phases, $\mathbf{c}_{g}(t)=\frac{\mathbf{n}_{g}(t)}{V_{g}(t)}$ and $\mathbf{c}_{l}(t)=\frac{\mathbf{n}_{l}(t)}{V_{l}(t)}$, are generated using Eqs. (2) and (3); $V_{l}(t)$ is calculated from the liquid density, which depends on the mixture composition, and $V_{g}(t)$ is inferred from $V_{r}-V_{l}(t)$. Zero-mean Gaussian noise with standard deviation corresponding to $1 \%$ of the maximal concentration of each species is added to $\mathbf{c}_{l}$ and $\mathbf{c}_{g}$. The concentrations given in Fig. 3 are measured every minute for one hour.

Since all concentrations in both phases are measured, the linear transformations proposed by [20] can be applied to transform the $S_{g}+S_{l}=2+5=7$ number of moles profiles to $R=2$ extents of reaction, $p_{m}=2$ extents of mass transfer and $p_{g}=1$ extent of inlet flow in the gas phase. The computed extents of reaction and mass transfer are shown in Fig. 4.

As all the transferring species involved in the liquid-phase reactions are included as gaseous species in the reaction scheme $\left(\mathrm{Cl}_{2(\mathrm{~g})}\right.$ and $\left.\mathrm{HCl}_{(g)}\right)$, Eq. (22) can be used to generate the heat flow signal $q_{r}$ with $u_{\text {out }}=0$ (semi-batch reactor) and the enthalpies of reaction $\Delta H_{r, 1}=-59.58 \mathrm{~kJ} \mathrm{~mol}^{-1}$ and $\Delta H_{r, 2}=-84.13 \mathrm{~kJ} \mathrm{~mol}^{-1}$. Zero-mean Gaussian noise with standard deviation corresponding to $3 \%$ of the maximal signal value is added to $q_{r}$. The computed extents of reaction $\mathbf{x}_{r}(t)$ and the integral heat signal $Q_{r}(t)$ are both used in Eq. (28) to estimate the reaction enthalpies. The estimated reaction enthalpies $\Delta \hat{H}_{r, 1}=-60.30$ (99\% C.I. $\left.=58.07-62.53\right) \mathrm{kJ} \mathrm{mol}^{-1}$ and $\Delta \hat{H}_{r, 2}=-79.41$ (99\% C.I. $\left.=59.65-99.16\right) \mathrm{kJ} \mathrm{mol}^{-1}$ are in very good agreement with the values used for the simulation. The broader confidence interval for the second estimated reaction enthalpy is due to the lower extent of the second reaction compared to the first reaction.

\section{Conclusions}

This paper has discussed ways of using concentration and calorimetric measurements in the context of extent based incremental identification of reaction systems. The use of calorimetric data has been shown to be particularly advantageous for two specific cases.

For certain reaction systems, it is not always possible to measure the concentration of all species. In such cases, when there are fewer measured species than the number of reactions and mass transfers, a calorimetric signal can be a valuable source of informa- 
tion for modeling purposes. A procedure for augmenting the rank of the measurement equations using calorimetric data so as to be able to compute the extents of reactions and mass transfer has been discussed. This involves adding the integral heat signal to the concentration measurements and, correspondingly, the reaction enthalpies to the stoichiometric matrix, and then use the reaction- and mass-transfer-variant form to compute the extents. The rate laws and the corresponding parameters can be identified based on computed extents. The acetoacetylation of pyrrole was simulated in order to demonstrate the feasibility of this rank augmentation. Rate expressions and rate parameters were identified from the extents computed from concentration and calorimetric measurements. For this application, the noise level in the heat signal does not play a major role, as shown by changing the noise level of the heat signal from $3 \%$ to $5 \%$.

Another application of calorimetry in the context of extentbased incremental identification is the estimation of reaction enthalpies from the computed extents and the measured heat signal. The chlorination of butanoic acid was simulated to illustrate the estimation of reaction enthalpies in a gas-liquid reaction system. Although reasonably accurate reaction enthalpies were predicted, robust estimation of enthalpies represents a difficult task, especially when the various extents of reaction are of different magnitude. This was observed for the case study of the chlorination of butanoic acid, where the small extent for the second reaction led to a broad confidence interval for the corresponding estimated reaction enthalpy.

\section{References}

[1] E. Walter, L. Pronzato, Identification of Parametric Models from Experimental Data, Springer-Verlag, Berlin, 1997.

[2] D.C. Montgomery, Design and Analysis of Experiments, sixth ed., John Wiley \& Sons, New York, 2005.

[3] Y. Bard, Nonlinear Parameter Estimation, Academic Press, New York, 1974.

[4] M.L. Brendel, Incremental Identification of Complex Reaction Systems. PhD thesis, RWTH Aachen, Germany, 2005.

[5] W. Marquardt, Model-based experimental analysis of kinetic phenomena in multi-phase reactive systems, Chem. Eng. Res. Des. 83 (A6) (2005) 561-573.
[6] N. Bhatt, N. Kerimoglu, M. Amrhein, W. Marquardt, D. Bonvin, Incremental identification of reaction systems - a comparison between rate-based and extent-based approaches, Chem. Eng. Sci., http://dx.doi.org/10.1016/ j.ces.2012.05.040.

[7] M.L. Brendel, D. Bonvin, W. Marquardt, Incremental identification of kinetic models for homogeneous reaction systems, Chem. Eng. Sci. 61 (16) (2006) 5404-5420.

[8] A. Bardow, W. Marquardt, Incremental and simultaneous identification of reaction kinetics: methods and comparison, Chem. Eng. Sci. 59 (13) (2004) 2673-2684.

[9] N. Bhatt, M. Amrhein, D. Bonvin, Incremental identification of reaction and mass-transfer kinetics using the concept of extents, Ind. Eng. Chem. Res. 50 (23) (2011) 12960-12974.

[10] N. Bhatt, Extents of Reaction and Mass Transfer in the Analysis of Chemical Reaction Systems. PhD thesis, No. 5028, EPFL, Lausanne, Switzerland, 2011.

[11] D.M. Price, Micro-thermal analysis and related techniques, in: M.E. Brown, P.K. Gallagher (Eds.), Recent Advances, Techniques and Applications, Handbook of Thermal Analysis and Calorimetry, vol. 5, Elsevier Science B.V., 2008, pp. 55-91 (Chapter 3).

[12] T.A. Godany, Y.M. Neuhold, K. Hungerbühler, Combined in situ monitoring method for analysis and optimization of the lithiation-fluoroacetylation of n(4-chlorophenyl)-pivalamide, Ind. Eng. Chem. Res. 50 (2011) 5983-5991.

[13] C. LeBlond, J. Wang, R.D. Larsen, C.J. Orella, A.L. Forman, R.N. Landau, J. Laquidara, J.R. Sowa Jr., D.G. Blackmond, Y.K. Sun, Reaction calorimetry as an in-situ kinetic tool for characterizing complex reactions, Thermochim. Acta 289 (2) (1996) 189-207.

[14] P.J. Haines, G.R. Heal, P.G. Laye, D.M. Price, S.B. Warrington, R. Wilson, in: Principles of Thermal Analysis and Calorimetry, The Royal Society of Chemistry, 2002, pp. 77-78.

[15] A. Zogg, F. Stoessel, U. Fischer, K. Hungerbühler, Isothermal reaction calorimetry as a tool for kinetic analysis, Thermochim. Acta 419 (1-2) (2004) 1-17.

[16] O. Ubrich, B. Srinivasan, P. Lerena, D. Bonvin, F. Stoessel, Optimal feed profile for a second-order reaction in a semi-batch reactor under safety constraints: experimental study, J. Loss Prev. Process Ind. 12 (6) (1999) 485-493.

[17] M. Amrhein, N. Bhatt, B. Srinivasan, D. Bonvin, Extents of reaction and flow for homogeneous reaction systems with inlet and outlet streams, AIChE J. 56 (2010) 2873-2886.

[18] A. Croce, The application of the concept of extent of reaction, J. Chem. Educ. 79 (4) (2002) 506

[19] M. Amrhein, Reaction and Flow Variants/Invariants for the Analysis of Chemical Reaction Data. PhD thesis, No. 1861, EPFL, Lausanne, Switzerland, 1998.

[20] N. Bhatt, M. Amrhein, D. Bonvin, Extents of reaction, mass transfer and flow for gas-liquid reaction systems, Ind. Eng. Chem. Res. 49 (2010) 7704-7717.

[21] G. Maria, A. Dan, D.N. Stefan, Model-based derivation of the safety operating limits of a semibatch reactor for the catalytic acetoacetylation of pyrrole using a generalized sensitivity criterion, Chem. Biochem. Eng. Q. 24 (3) (2010) 265281. 